\title{
CONVERGENCE OF THE SCHUR ALGORITHM
}

\author{
OLAV NJÅSTAD
}

(Communicated by Paul S. Muhly)

\begin{abstract}
Convergence of the Schur algorithm outside the unit circle is discussed with the aid of convergence theory for Schur continued fractions.
\end{abstract}

\section{INTRODUCTION}

In [6] Schur introduced and discussed what is now called the Schur algorithm. This algorithm generates from a sequence $\left\{\gamma_{n}\right\}$ of parameters a sequence $\left\{F_{n}(z)\right\}$ of rational functions. Under suitable positivity conditions, the functions $F_{n}(z)$ are Schur functions, i.e., functions which are analytic and bounded by the constant 1 in the open disk $D=\{z \in \mathbf{C}:|z|<1\}$; and the sequence $\left\{F_{n}(z)\right\}$ converges for $z \in D$ to a Schur function $f(z)$. (The parameters $\gamma_{n}$ may in turn originate from a Schur function. For a general description, see $\S 2$.)

Schur illustrated the working of the algorithm by briefly sketching a few examples [6, p. 144]. We shall mention the two main ones later, here called Example 1 and Example 2 (see $\S 4$ ). In both these examples, the sequence $\left\{\gamma_{n}\right\}$ satisfies the additional condition $\sum_{n=1}^{\infty}\left|\gamma_{n}\right|^{2}<\infty$. In [5, p. 106] Runckel observed that in the case of Example 1, the sequence $\left\{F_{n}(z)\right\}$ also converges in the outside domain $E=\mathbf{C}-\bar{D}=\{z \in|z|>1\}$ to an analytic function.

In this paper we discuss (under the restriction $\sum_{n=1}^{\infty}\left|\gamma_{n}\right|^{2}<\infty$ ) a necessary and sufficient condition for convergence of $\left\{F_{n}(z)\right\}$ in $E$ to take place, and point out that such convergence also occurs in Example 2. In our treatment we make use of results on convergence of special continued fractions closely related to the Schur algorithm: Schur fractions. The theory of these continued fractions is in turn closely related to the theory of Perron-Carathéodory fractions and of Szegö polynomials, and our condition for convergence may be stated in terms of asymptotic behavior of Szegö polynomials.

For general theory of continued fractions we refer to [3]. For discussion of the Schur algorithm we refer to [6], and also to [1, 5, 7]. The theory of Schur fractions is discussed, e.g., in [1], [3], [4], [7], and the theory of Perron-

Received by the editors August 28, 1989.

1980 Mathematics Subject Classification (1985 Revision). Primary 30E05, 42 C05.

Key words and phrases. Schur algorithm, Schur continued fractions. 
Carathéodory fractions and their relationship to Szegö polynomials, e.g., in [1], [2], [4].

\section{The SChUR ALgorithm AND Schur FRActions}

The Schur algorithm may be defined as follows. Let $\left\{\gamma_{n}: n=0,1,2, \ldots\right\}$ be a sequence of complex numbers where $\left|\gamma_{n}\right| \neq 1$ for all $n$. Let $z \in \mathbf{C}$, and set

$$
\begin{aligned}
t_{n}(z, w) & =\frac{\gamma_{n}+z w}{1+\bar{\gamma}_{n} z w} \text { for } n=0,1,2, \ldots \\
T_{0}(z, w) & =t_{0}(z, w), \\
T_{n}(z, w) & =T_{n-1}\left(z, t_{n}(z, w)\right) \text { for } n=1,2, \ldots
\end{aligned}
$$

The approximants of the algorithm are defined as the rational functions

$$
F_{n}(z)=T_{n}(z, 0) \text {. }
$$

Convergence of the algorithm means convergence of the approximants.

The sequence $\left\{\gamma_{n}\right\}$ also gives rise to a Schur fraction. This is a continued fraction of the form

$$
\gamma_{0}+\frac{\left(1-\left|\gamma_{0}\right|^{2}\right) z}{\bar{\gamma}_{0} z}+\frac{1}{\gamma_{1}}+\frac{\left(1-\left|\gamma_{1}\right|^{2}\right) z}{\bar{\gamma}_{1} z}+\cdots+\frac{1}{\bar{\gamma}_{n}}+\frac{\left(1-\left|\gamma_{n}\right|^{2}\right) z}{\bar{\gamma}_{n} z}+\cdots
$$

We denote the numerators and denominators of (2.4) by $A_{n}(z)$ and $B_{n}(z)$. These functions satisfy the recurrence relations

$$
\left(\begin{array}{l}
A_{2 n} \\
B_{2 n}
\end{array}\right)=\gamma_{n}\left(\begin{array}{l}
A_{2 n-1} \\
B_{2 n-1}
\end{array}\right)+\left(\begin{array}{l}
A_{2 n-2} \\
B_{2 n-2}
\end{array}\right), \quad n=1,2, \ldots
$$

The polynomials $A_{2 n}, B_{2 n}$ are of degree at most $n$, while the polynomials $A_{2 n+1}, B_{2 n+1}$, are of degree at most $n+1$ with constant term zero.

The even and odd numerators and denominators are related as follows:

$$
\begin{array}{ll}
A_{2 n+1}(z)=z^{n+1} \overline{B_{2 n}}\left(\frac{1}{z}\right), & B_{2 n+1}(z)=z^{n+1} \overline{A_{2 n}}\left(\frac{1}{z}\right) \\
A_{2 n}(z)=z^{n+1} \overline{B_{2 n+1}}\left(\frac{1}{z}\right), & B_{2 n}(z)=z^{n+1} \overline{A_{2 n+1}}\left(\frac{1}{z}\right)
\end{array}
$$

(where the bar denotes complex conjugation of coefficients). It follows that

$$
\frac{A_{2 n+1}(z)}{B_{2 n+1}(z)}=\left(\left(\overline{\frac{A_{2 n}\left(\frac{1}{z}\right)}{B_{2 n}\left(\frac{1}{z}\right)}}\right)\right)^{-1}, \quad \frac{A_{2 n}(z)}{B_{2 n}(z)}=\left(\left(\overline{\frac{A_{2 n+1}\left(\frac{1}{z}\right)}{B_{2 n+1}\left(\frac{1}{z}\right)}}\right)\right)^{-1} .
$$


The even approximants of the Schur fraction coincide with the approximants of the Schur algorithm, i.e.,

$$
F_{n}(z)=T_{n}(z, 0)=\frac{A_{2 n}(z)}{B_{2 n}(z)} .
$$

A Schur fraction is called positive if

$$
\gamma_{0} \in \mathbf{R}, \quad\left|\gamma_{n}\right|<1 \text { for } n=0,1,2, \ldots
$$

In this case the approximants $F_{n}(z)=A_{2 n}(z) / B_{2 n}(z)$ are Schur functions.

Every Schur function $f(z)$ gives rise to a sequence $\left\{f_{n}(z)\right\}$ of Schur functions and a parameter sequence $\left\{\gamma_{n}\right\}$ generating a positive Schur fraction as follows:

$$
\begin{aligned}
f_{0}(z)=f(z), \quad f_{n+1}(z) & =\frac{f_{n}(z)-\gamma_{n}}{z\left(1-\bar{\gamma}_{n} f_{n}(z)\right)} \text { for } n=0,1,2, \ldots \\
\gamma_{n} & =f_{n}(0) .
\end{aligned}
$$

Let $t_{n}, T_{n}$ be the linear transformations defined by the parameter sequence $\left\{\gamma_{n}\right\}$. Then

$$
f_{n}(z)=t_{n}\left(z, f_{n+1}(z)\right), \quad f(z)=T_{n}\left(z, f_{n+1}(z)\right) .
$$

For more detailed discussions, see especially $[1$, p. 129-136; 5, p. 97-99; 6, p. 137-140].

\section{Convergence Results}

In this section we shall first mention some known results on convergence of even and odd approximants of a positive Schur fraction. For proofs and further details, see $[1,4,5,6]$.

Let (2.4) represent a positive Schur fraction with $\gamma_{0} \neq 0$, i.e.,

$$
\gamma_{0} \in \mathbf{R}-\{0\}, \quad\left|\gamma_{n}\right|<1 \quad \text { for } n=0,1,2, \ldots
$$

Then the even approximants $A_{2 n}(z) / B_{2 n}(z)$ (which are Schur functions) converge for $z \in D$ (uniformly on compact subsets) to a Schur function $f(z)$, which gives rise to the parameter sequence $\left\{\gamma_{n}\right\}$ via (2.10)-(2.11) (see e.g. [1, p. 134]). It follows that the odd approximants $A_{2 n+1}(z) / B_{2 n+1}(z)$ converge for $z \in E$ to the function $\left(\bar{f}\left(\frac{1}{z}\right)\right)^{-1}$.

Assume that in addition to (3.1), the following condition is also satisfied:

$$
\sum_{n=0}^{\infty}\left|\gamma_{n}\right|^{2}<\infty
$$

Then the even numerators $A_{2 n}(z)$ and denominators $B_{2 n}(z)$ converge separately for $z \in D$ to analytic functions $A(z)$ and $B(z)$, where $B(z) \neq 0$ for $z \in D$. Furthermore the odd numerators $A_{2 n+1}(z)$ and denominators $B_{2 n+1}(z)$ converge to zero for $z \in D$. It follows that $z^{-(n+1)} A_{2 n+1}(z)$ and 
$z^{-(n+1)} B_{2 n+1}(z)$ converge to $\bar{B}\left(\frac{1}{z}\right)$ and $\bar{A}\left(\frac{1}{z}\right)$ for $z \in E$, while $z^{-(n+1)} A_{2 n}(z)$ and $z^{-(n+1)} B_{2 n}(z)$ converge to zero for $z \in E$. See e.g. [4, p. 99-106].

We shall now prove a result concerning convergence of the odd approximants $A_{2 n+1}(z) / B_{2 n+1}(z)$ for $z \in D$, or equivalently of the even approximants $A_{2 n}(z) / B_{2 n}(z)$ for $z \in E$. The relevant condition is in terms of asymptotic behavior of the $(n+1)$ th degree polynomials $B_{2 n+1}(z)$ for $z \in D$. More precisely, in terms of the limiting behavior of the quotient, $B_{2 n+1}(z) / z^{n+1}$ for $z \in D$. (The condition may easily be translated into a condition on asymptotic behavior of the Szegö polynomials determined by the parameters $\left\{\gamma_{n}\right\}$, as indicated in $\S 1$.)

Theorem. Assume that the sequence $\left\{\gamma_{n}\right\}$, in addition to satisfying (3.1) and (3.2), also is such that

$$
\lim _{n \rightarrow \infty} \frac{z^{n+1}}{B_{2 n+1}(z)}=0 \quad \text { for } z=D .
$$

Then the following hold:

$$
\text { I. } \quad \lim _{n \rightarrow \infty} \frac{A_{2 n+1}(z)}{B_{2 n+1}(z)}=\frac{A(z)}{B(z)}=f(z) \text { for } z \in D \text {, }
$$

$$
\text { II. } \quad \lim _{n \rightarrow \infty} F_{n}(z)=\lim _{n \rightarrow \infty} \frac{A_{2 n}(z)}{B_{2 n}(z)}=\left(\bar{f}\left(\frac{1}{z}\right)\right)^{-1} \text { for } z \in E \text {. }
$$

Condition (3.3) is also necessary for I and II to hold.

Proof. Statements I and II are equivalent by (2.7). We shall prove I.

We may write

$$
\frac{A_{2 n+1}(z)}{B_{2 n+1}(z)}-f(z)=\left(\frac{A_{2 n+1}(z)}{B_{2 n+1}(z)}-\frac{A_{2 n}(z)}{B_{2 n}(z)}\right)+\left(\frac{A_{2 n}(z)}{B_{2 n}(z)}-f(z)\right) .
$$

The second term of (3.6) tends to zero, since (3.1) is satisfied. By using the determinant formula for continued fractions (see [3, p. 20]) we may write the first term as follows:

$$
\frac{A_{2 n+1}(z)}{B_{2 n+1}(z)}-\frac{A_{2 n}(z)}{B_{2 n}(z)}=\frac{z^{n+1} \prod_{k=0}^{n}\left(1-\left|\gamma_{k}\right|^{2}\right)}{B_{2 n+1}(z) B_{2 n}(z)} .
$$

Again since (3.1)-(3.2) are satisfied, $\lim _{n \rightarrow \infty} B_{2 n}(z)=B(z)$ for $z \in D$, where $B(z) \neq 0$. The product $\prod_{k=0}^{n}\left(1-\left|\gamma_{k}\right|^{2}\right)$ is bounded, and tends to a finite limit different from zero. Condition (3.3) then ensures that the right side of (3.7) tends to zero, and vice versa. This completes the proof.

\section{EXAMPLES}

In both of the examples given below, (3.1)-(3.2) are clearly satisfied and by using explicit formulas for $A_{n}(z), B_{n}(z)$ given in [1, p. 147-150], it can be 
seen through calculation that (3.3) is satisfied. Hence $A_{n}(z) / B_{n}(z)$ converges for $z \in D$. This can also, by using the above-mentioned formulas, be verified directly.

Example 1. Set $\gamma_{n}=1 /(n+2), n=0,1,2, \ldots$. Here

$$
\lim _{m \rightarrow \infty} \frac{A_{2 m}(z)}{B_{2 m}(z)}=\frac{1}{2-z} \text { for } z \in D
$$

(see [1, p. 148], [6, p. 144]), and thus

$$
\lim _{m \rightarrow \infty} \frac{A_{2 m}(z)}{B_{2 m}(z)}=2-\frac{1}{z} \quad \text { for } z \in E .
$$

Example 2. Set $\gamma_{0}=\frac{1}{2}, \gamma_{n}=\frac{2}{2 n+1}, n=1,2, \ldots$ Here

$$
\lim _{m \rightarrow \infty} \frac{A_{2 m}(z)}{B_{2 m}(z)}=\frac{1}{2}(1+z) \quad \text { for } z \in D ;
$$

hence

$$
\lim _{m \rightarrow \infty} \frac{A_{2 m}(z)}{B_{2 m}(z)}=\frac{2 z}{z+1} \quad \text { for } z \in E .
$$

\section{REFERENCES}

1. W. B. Jones, O. Njästad, and W. J. Thron, Schur fractions, Perron-Carathéodory fractions and Szegö polynomials, a survey, in Analytic Theory of Continued Fractions II (W. J. Thron, ed.), Lecture Notes in Math., vol. 1199, Springer-Verlag, New York, 1986, pp. 127-158.

2. __ Moment theory, orthogonal polynomials, quadrature, and continued fractions associated with the unit circle, Bull. London Math. Soc. 21 (1989), 113-152.

3. W. B. Jones and W. J. Thron, Continued fractions: analytic theory and applications, Encyclopedia of Mathematics and its Applications, vol. 11, Addison-Wesley, 1980 (distributed now by Cambridge University Press).

4. $\mathrm{O}$. Njảstad, A survey of some results on separate convergence of continued fractions, in Analytic Theory of Continued Fractions III (L. Jacobsen, ed.), Lecture Notes in Math., vol. 1406, Springer-Verlag, New York, 1989, pp. 88-114.

5. H-J. Runckel, Bounded analytic functions in the unit disk and the behavior of certain analytic continued fractions near the singular line, J. Reine Angew. Math. 281 (1976), 97-125.

6. I. Schur, Über Potenzreihen, die im Innern des Einheitskreises beschränkt sind, J. Reine Angew. Math. 147 (1916), 205-232; 148 (1917), 122-145.

7. H. S. Wall, Continued fractions and bounded analytic functions, Bull. Amer. Math. Soc. $\mathbf{5 0}$ (1944), 110-119.

Department of Mathematics, University of Trondheim-NTH, N-7034 Trondheim, NORWAY 